H. Fischer, A. Endmann \& M. Krökel (Hrsg.): Mensch und Computer 2015 - Usability Professionals, Berlin: De Gruyter, S. 252- 260.

\title{
Reminder Objects
}

\section{Greifbare Interaktion im alltäglichen Leben}

\author{
Henrik Rieß \\ User Interface Design $\mathrm{GmbH}$ \\ Rankestraße 8 \\ 10789 Berlin \\ henrik.riess@uid.com

\section{Martin Ecker} \\ User Interface Design $\mathrm{GmbH}$ \\ Wilhelm-Bleyle-Str. 10-12 \\ 71636 Ludwigsburg \\ martin.ecker@uid.com
}

\author{
Dr. Peter Klein \\ User Interface Design $\mathrm{GmbH}$ \\ Rankestraße 8 \\ 10789 Berlin \\ peter.klein@uid.com \\ Stefan Hintz \\ User Interface Design $\mathrm{GmbH}$ \\ Rankestraße 8 \\ 10789 Berlin \\ stefan.hintz@uid.com
}

\begin{abstract}
Reminder Objects bezeichnen Alltagsgegenstände, die mit digitalen Informationen vernetzt sind, und durch ihr objekthaftes Verhalten Nutzer bei wichtigen Entscheidungen und Hilfegesuchen unterstützen. Unter Objekthaftigkeit sind in diesem Falle alle physischen Merkmale eines Produktes zu verstehen, die unsere Sinne erfassen können.
\end{abstract}

Der Beitrag erklärt das Konzept der Reminder Objects, zeigt auf, wie man diese entwickelt, und stellt Projektbeispiele vor.

\section{Keywords}

Internet der Dinge, Internet of Things, Reminder Objects, Interaction Design, User Experience, Tangible Interaction, Physical Computing

Sei es der Blick auf den Wetterbericht („Muss ich mich auf Schmuddelwetter einstellen?“), der Weg zum nächsten Notdienst (,Wohin muss ich gehen, um jemanden zu finden, der mir weiterhilft?“) oder die Frage nach dem Kontostand am Monatsende (,Kann ich mir diese Sneakers noch leisten?"): Diese Informationen sind im Internet vorhanden, doch gerade in zeitkritischen Situationen und im mobilem Kontext ist das Suchen nach ihnen stressig und unkomfortabel.

Unterwegs wird aktuell vor allem über das Display (Smartphones, Laptops, Anzeigen im öffentlichen Raum) auf digitale Informationen zugegriffen. Die Informationsaufnahme läuft vorrangig über den visuellen Sinn. Zweitranging wird dann oft die Akustikebene 
eingebunden (Durchsagen, Töne). Viele der zu betrachtenden Screens sind durch ihre physikalischen Abmessungen zu klein, um die Information mit allen Zusammenhängen detailliert darzustellen. Dazu kommen physische Einschränkungen von Nutzern wie Weitsichtigkeit, fehlende Mobilität oder psychische Faktoren wie die Angst, sensible Daten in der Öffentlichkeit aufzurufen.

So fand UID im BMBF-Forschungsprojekt inDAgo in Fokusgruppen und Kontextinterviews heraus, dass Senioren Ausflüge in ihrem Wohnort vermieden. Der Grund: Sie fühlten sich von der Fülle an Fahrplaninformationen sowie Änderungen an Aushängen und in mobilen Applikationen überfordert und hatten Angst, nicht wieder zurück zu finden. Das Gefühl der Ohnmacht, mit dem falschen Ticket in die falsche Bahn einzusteigen, lässt sie dann lieber gar nicht ausgehen. Dabei würde oft schon der Hinweis reichen, auf dem richtigen Weg zu sein.

Führt man diesen Gedanken weiter, so genügt anstelle des Scrollens durch Ergebnislisten oft bereits eine weniger präzise, aber eindeutige Information, um eine sichere Entscheidung treffen zu können.

Ansatz der Reminder Objects ist es, Gegenstände unserer Umgebung zu identifizieren, die tägliche Handlungsabläufe unterstützen, diese mit hilfreichen Informationen zu verknüpfen und als Entscheidungshilfen und Erinnerungen in der Interaktion zwischen Objekt und Nutzer zugänglich zu machen.

\section{Internet der alltäglichen Dinge}

In seinem Buch „Die Dinge des Alltags“ (1989: 11f.) spricht Donald Norman von ca. zwanzig- bis dreißigtausend Gegenständen, auf die wir im Alltag stoßen. Angefangen von der morgendlichen Tortur des Wecker, dem Bürostuhl, mit dem wir einige hunderte Male auf dem Linoleum scharren, bis hin zum Schlüssel, der uns Abends wieder die Wohnungstür öffnet.

Ein besonders gutes Beispiel für rein analoge Reminder Objects ist das Wetterhäuschen, das viele vielleicht noch aus ihrer Kindheit kennen. Relativ verlässlich zeigt das kleine Haus an, wie sich das Wetter der nächsten Stunden entwickelt. Einmal erlernt, weiß jedes Kind das Häuschen zu interpretieren, ohne die Funktionsweise des dahinter steckenden Hygrometers zu kennen: Die Sonnenfrau bringt gutes Wetter, beim Regenmann dagegen sollte der Schirm für unterwegs nicht fehlen. Beide Figuren sind auf einer Drehscheibe angebracht, die von einer Feder in eine Richtung gezogen wird. Auf der Gegenseite dagegen sitzt ein Stück tierisches Material (bspw. Pferdehaar), welches sich bei Feuchtigkeit ausdehnt oder bei Trockenheit zusammenzieht.

Digitale Informationen liegen oftmals in hohem Detailgrad vor und ermöglichen - richtig interpretiert - ein wesentlich weitsichtigeres Reagieren. Für die Visualisierung persönlicher Informationen und Entscheidungsvorlagen fehlt es jedoch jenseits der Smartphone-Screens 
noch weitestgehend an klaren Anzeigeformaten. Daher sind Wearables der nächste Schritt zu einer vernetzten, reaktionsfähigen Umgebung mit Sensoren und Aktoren.

Die daraus resultierende Frage ist also: Wie sehen die „Wetterhäuschen“ aus, die den Surplus des Internets nutzen? Und wie lassen sich die Werte in physisches Objektverhalten transformieren, das sich im Kontext des Auftretens intuitiv erschließt?

\section{I/O: Aufforderung an unsere Sinne}

Für die nachfolgenden Forschungsbeispiele lag der Ansatz darin, In- und Output von Technologien mit denen menschlicher Wahrnehmung abzugleichen. Ziel war es, mindestens einen Sinn vordergründig oder sogar mehrere Sinne anzusprechen und Informationsveränderungen über eine Veränderung der Objekteigenschaften sensorisch (be)greifbar zu machen.

Die klassischen Sinneskanäle dabei sind Sehen (Visuell - Farbe und Helligkeit), Hören (Auditiv - Tonhöhe und Lautstärke), Riechen (Olfaktorisch - Geruch), Schmecken (Gustatorisch - Geschmack) und Fühlen (Taktil - Druck, Berührung, Vibration). Nach aktueller Betrachtung verfügt der Mensch noch weitere, stimulierbare Sinne (vgl. Physiologie des Menschen, Schmidt \& Thews, 1997): Gleichgewichtssinn (Körperhaltung und Orientierung im Raum), Temperatursinn (Wärme und Kälte) sowie den vestibulären Sinn (Beschleunigung).

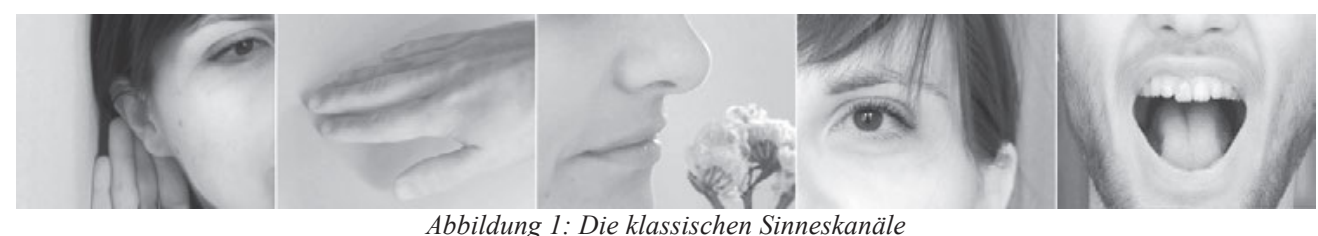

Abbildung 1: Die klassischen Sinneskanäle

Da jeder Mensch sowohl physiologisch als auch psychologisch über seine Sinnesebenen einen individuellen Zugang zu Kommunikation hat, ist es förderlich, wenn die Interaktion mit dem System eine breite Anzahl an Modalitäten unterstützt, so dass ein Austausch von Informationen eindeutig und situativ passend stattfindet. Die Sinneskanäle können einzeln voneinander und dramaturgisch miteinander verknüpft angesprochen werden.

In den nachfolgenden Forschungsbeispielen haben wir uns weniger auf die Entscheidung des Nutzers für eine bestimmte Modalität aus mehreren fokussiert, sondern haben für einzelne Szenarios experimentell ermittelt, wie analog-digitale Interfaces über Wahrnehmungsebenen kommunizieren und in entsprechenden Situationen auf sich aufmerksam machen.

Situativ eingesetzt, enthält jeder Impuls, der vom Reminder Object ausgeht, eine sensorische Affordanz. Diese zielt auf die entsprechende Modalität des Nutzers (Sehen, Hören, Fühlen...), wird kognitiv zuordnet und in (ggf. rückgekoppelte) Entscheidungen umsetzt. 
Beispiel: Die Erkenntnis, dass meine Jacke an meiner rechten Schulter vibriert, weil sie mich zu einer Richtungsänderung bewegen möchte.

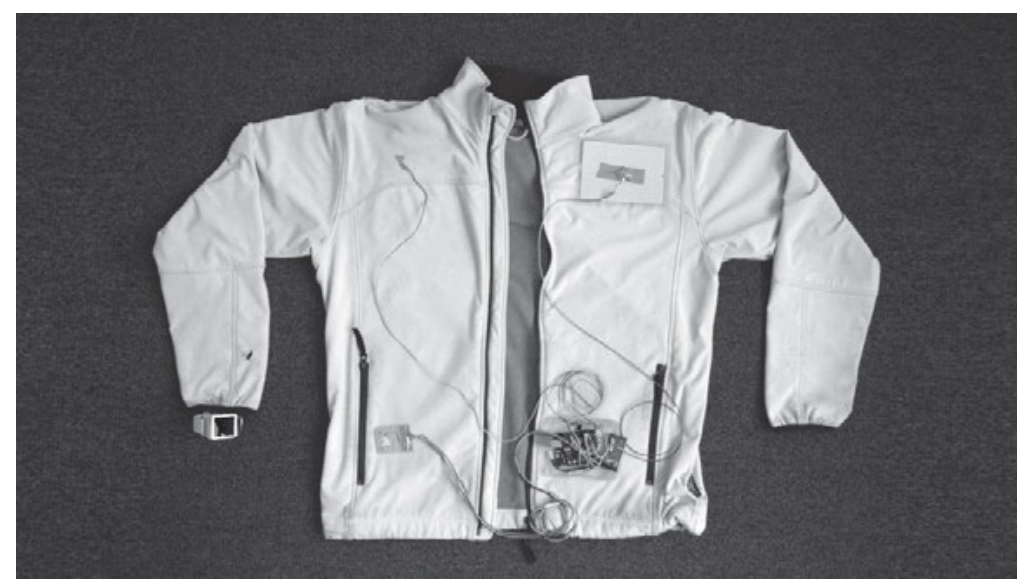

Abbildung 2: Jacke „Spirit Guide“ aus dem Forschungsprojekt inDAgo

Für bestehende Alltagsobjekte bedeutet dies, dass neben dem offensichtlichen Angebotscharakter - im Falle der Jacke das Ankleiden - ein zweiter, verborgener hinzukommt. Somit ist die Jacke nicht nur Kleidungsstück, sondern auch vertrauensvoller Wegweiser.

Für Gestalter tun sich damit in Zeiten der Implementierung des Internet of Things riesige Potentiale, aber auch Risiken auf, wenn es darum geht, das Verhalten analog-digital koexistierender uns bislang vertrauter Gegenstände mitzugestalten.

\section{Theorie: Die Triple-6-Methode}

Dies ist eine Abwandlung der 6-3-5 Methode, welche im Brainwriting angewandt wird, um Ideen zu finden. Bei der klassischen 6-3-5 Methode erhalten sechs Teilnehmer ein Blatt mit sechs Zeilen und drei Spalten. Jeder Teilnehmer schreibt drei Ideen auf. Anschließend wird das Blatt weitergereicht. Der nächste Teilnehmer greift die Ideen des Vorgängers auf und entwickelt sie weiter. Bei der Tripel6-Methode sind die Teilnehmeranzahl und Runden gleich wie bei der 6-3-5 Methode. Lediglich die Anzahl der Ideen ist nicht auf 3 begrenzt stattdessen, werden den Spalten verwendbare Modalitäten zugeordnet (z. B. Sehen, Hören, Riechen, Fühlen, Beschleunigung und Temperatur spüren).

Jeder Teilnehmer formuliert in der ersten Spalte je eine Idee für einen Sinneskanal. Danach werden die Blätter im Urzeigersinn zum Nächsten weitergereicht, der die Idee aufgreift und weiterentwickelt. Nach einem Durchlauf steht somit eine große Anzahl theoretischer Ansätze zur Verfügung, die später im Experiment mit Technologie prototypisch erfahrbar gemacht und ausgeschlossen oder weiterentwickelt werden können. 
Vorteil: Aus Gewohnheit sind digitale Gestalter oft geneigt, zunächst mit visuellen Reizen zu arbeiten, danach unterstützend mit akustischen und taktilen Signalen, die gerade im mobilen Kontext sehr überzeugen können. Insofern zwingt diese Erweiterung der Methode zu einer sehr differenzierten Betrachtung der Gestaltung einzelner Modalitäten und führt zu disruptiven Ideen.

Kritik: Nicht alle Modalitäten lassen sich in jeder Situation gleich bedienen. Andere machen z.T. wenig Sinn und können schon theoretisch ausgeschlossen werden.

\section{Praxis: Das Experiment}

Bei allen Projektbeispielen, die sich an unsere Sinne wenden, eignen sich Kreativmethoden wie die Triple-6-Methode für den Beginn der Ideenphase hervorragend, um erste Produktideen zu generieren. Gerade zu Beginn des Gestaltungsprozesses braucht es aber noch einen Gegenimpuls, welcher Sehen, Anfassen, Hören... direkt in Form von Technologie erlebbar macht.

Hier sollte eine strukturell-experimentelle Entwicklungsphase ganz zu Beginn des klassischen User-Centered-Design-Prozesses einbezogen werden. In dieser provoziert die Konfrontation aus der emotionalen Ansprache der Nutzer und einer medienadäquaten Ausschöpfung technischer Artefakte eine neue Sicht auf Lösungswege und Nutzung bereits vorhandener Ressourcen. Gestalter sollten sich dabei lediglich von der Frage leiten lassen „Wie kann diese Technologie vielleicht unseren Use Case unterstützen?“ Eine Kinect kann beispielsweise neue Ideen für den Einsatz von Gesichtserkennung geben. Ebenso kann ein selbstgebauter Drucksensor über mehrere Punkte auf einer Fläche verteilt einen Präsenzsensor ersetzen.

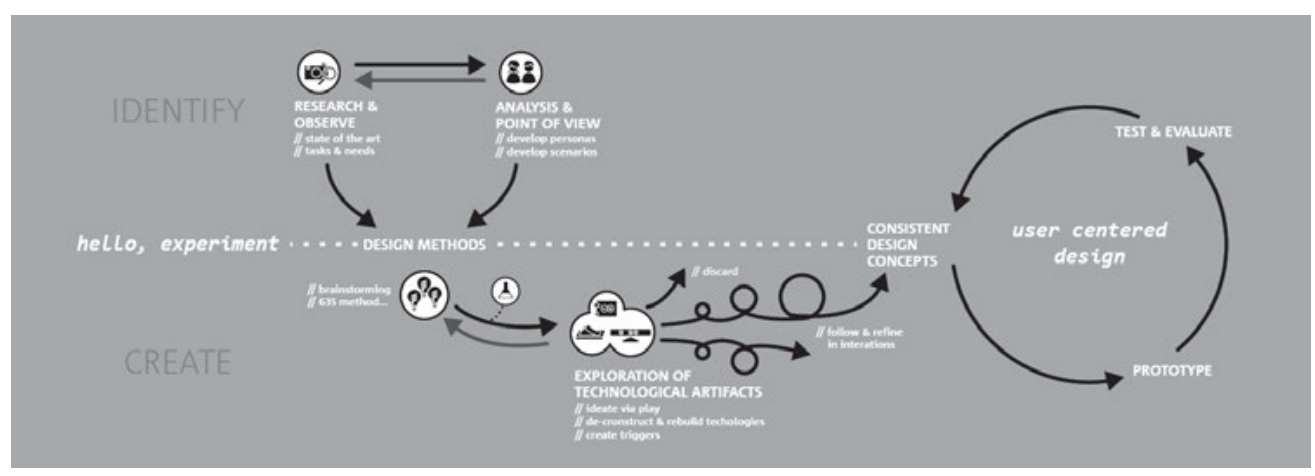

Abbildung 3: Experiment

Vorteil: Überzeugende Lösungen können schnell identifiziert werden, während faule Kompromisse ebenso schnell für die weitere Gestaltung ausgeschlossen werden können. 
Im Gegensatz zu rein ingenieursbezogenen Designprozessen mit Prototypenbau ist der Technologietransfer im strukturell-experimentellen Prozess weniger Proof of Concept, sondern mehr Möglichkeit, für Gestalter einen kreativen Kontrapunkt zu vermeintlich eindeutigen Produktanforderungen zu entwickeln.

\section{Beispiel 1 - Spirit Guide}

Spirit Guide wurde im Kontext des BMBF-Forschungsprojektes inDAgo entwickelt, welches sich mit Gestaltungslösungen beschäftigt, die älteren Leuten bei ihren täglichen Problemen im öffentlichen Personennahverkehr der Städte helfen soll.
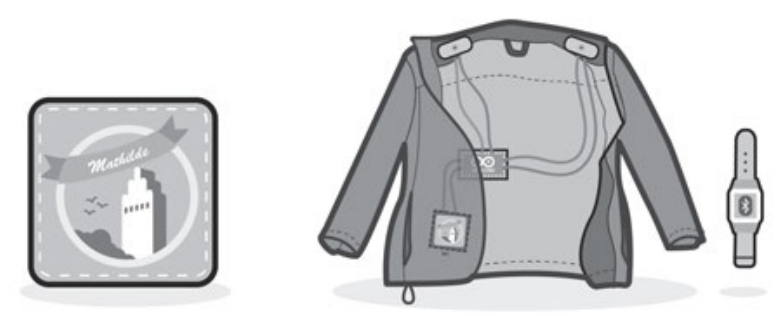

Abbildung 4: Jacke „Spirit Guide“ und Location Badget

Innerhalb des prototypischen Szenarios „Finden statt Suchen“ wurde eine handelsübliche Jacke mit dem Internet verbunden. Auf einer der Jackentaschen befindet sich eine Klettfläche, auf der „Location Badges“ angebracht werden können. „Location Badges“ sind kleine, flache Abzeichen, die einen NFC-Chip (Near Field Communication) besitzen. Jedes Badge besteht aus einem einzigartigen, gestickten Motiv und repräsentiert eine Person oder einen Ort.

Die Badges können Informationen, wie die GPS-Koordinate eines Ortes, speichern. Diese Informationen können reale Orte repräsentierten (wie „Bringe mich nach Hause“) oder einen flexiblen Point-of-Interest (wie „Führe mich zur nächstgelegenen Apotheke“) beschreiben. Die Klarheit und Selbstbeschreibungsfähigkeit machen die „Location Badges“ zu einer einfachen und intuitiven Eingabemethode für Personen jeden Alters.

Sobald der Location Badge auf den Klettkontakt gedrückt wird, übermittelt der Badge die enthaltenden Koordinaten via NFC drahtlos an ein konnektives Arduino-basiertes Navigationssystem, welches in die Jackeninnentasche eingenäht ist. Die Interaktion mit dem Träger der Jacke erfolgt über vibrierende Schulterpolster, die dem Nutzer mit haptischem Feedback den Weg weisen und ihn über plötzliche Routenänderungen informieren. Details wie Verspätungen und alternativen Routen lassen sich wiederum rückgekoppelt am Screen von Handy oder Smartwatch einsehen. 


\section{Beispiel 2 - Rainminder}

Rainminder ist eine prototypische Installation für den Eingangsbereich von Wohnungen. Für das Szenario „das Haus verlassen“ entstand die Idee einer mit dem Internet verbundenen Fußmatte, die beim Betreten informiert, ob es im Laufe des Tags Regenwetter gibt und den Nutzer beim Rausgehen gegebenenfalls erinnert, einen Schirm mitzunehmen.

Unabhängig vom Alter sollte die Installation gleichermaßen für Schulkinder, berufstätige Erwachsene und Senioren funktionieren, indem die Information spielerisch, aber gezielt über sensorische Affordanzen vermittelt wird.

Für die sinnlich erfassbare Darstellung des Regens boten sich folgende Modalitäten an:

- Sehen - Visuell - Projektion einer Regensilhouette; Visualisierung auftürmender Wolken

- Hören - Auditiv - Regentropfen, Pfützen, Donnern

- Riechen - Olfaktorisch - Geruch eines Sommerregens

- Fühlen - Taktil - Sprühnebel winziger Regentropfen auf der Haut

Für den ersten Prototyp der Fußmatte wurde sich auf die auditive Affordanz fokussiert: Bei Regenwetter klingt jeder Schritt auf der Matte wie der Sprung in eine Regenpfütze. Bei sonnigem Wetter dagegen bleibt die Matte still.
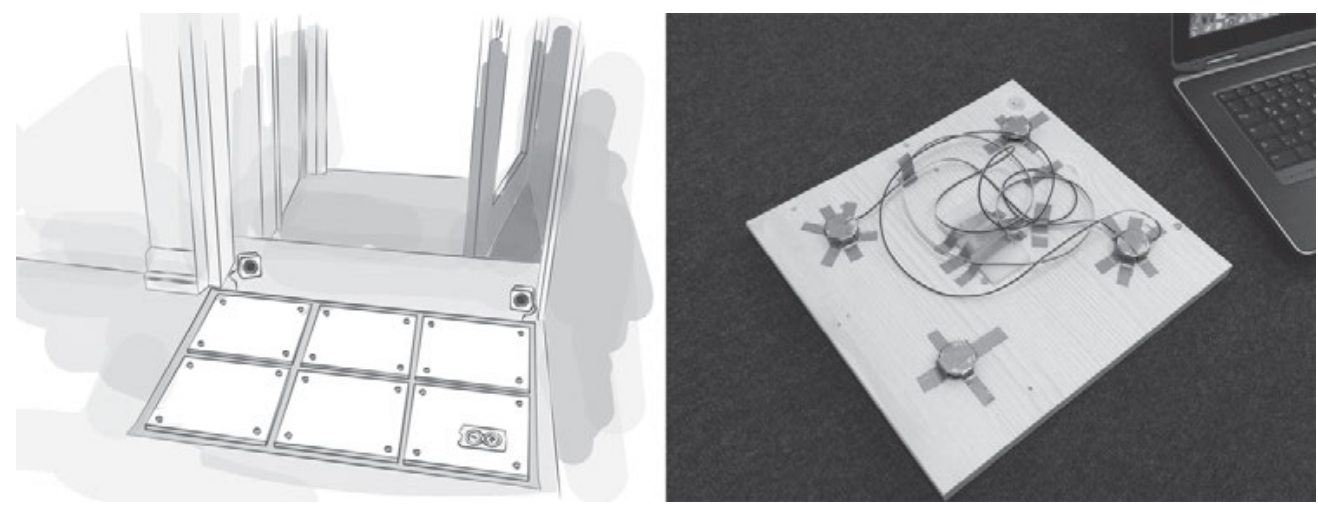

Abbildung 5: Rainminder

Der erste Prototyp basiert auf Holzplatten, die an Unterseiten mit Drucksensoren ausgestattet sind. Betritt eine Person die Platte, ändert sich der Widerstand der Sensoren und ein Signal wird an ein verbundenes Arduino Yún Board gesendet. Das Board interpretiert die Werte der Sensoren und stellt fest, dass ein Nutzer die Matte betreten hat.

Daraufhin fragt es die aktuelle Wettervorhersage für den aktuellen Ort über die API von openweathermap.org ab. Meldet die Wettervorhersage Niederschlag für den aktuellen Tag, so spielt das Board über angeschlossene Lautsprecher einen randomisierten Sound ab, der an 
den Tritt in eine Wasserpfütze erinnert. So ergibt sich für den Nutzer das Gefühl, als würde er an einem regnerischen Tag durch Wasserpfützen laufen. Aus der akustischen Affordanz heraus kann der Nutzer noch schnell zu Schirm oder Friesennerz greifen.

Ein realistischer Effekt ergibt sich, wenn die Sensordaten mehrerer Platten ausgewertet werden, so dass bei jedem Schritt des Nutzers ein Sound hörbar ist. Ebenso beeinflusst die Intensität des Auftritts die Charakteristik des wiedergegebenen Sounds.

\section{Beispiel 3 - BESTÅBOOTH}

BESTÅBOOTH ist eine Hommage an den klassischen Berliner Fotoautomaten (http://www.photoautomat.de/). Die alten Kabinen aus den 70er Jahren sind ein Tummelort für junge Menschen geworden, die gemeinsam ein Erlebnis teilen und in einem $\mathrm{S} / \mathrm{W}$-Abzug festhalten wollen.

Für das experimentelle Projekt BESTÅBOOTH wurde das Phänomen der selbsttätig arbeitenden Box analysiert und ein prototypisches Gestaltungskonzept für das Jahr 2015 entwickelt. Der Schlüssel des Erlebnisses „Automat“ ist die Unbedarftheit des Interfaces: Es gibt keine Buttons, keine Touchscreens. Als Input des Nutzers genügt der Einwurf eines 2Euro-Stückes. Dann läuft der Countdown, bis die 4er Aufnahmereihe erfolgt. Eine halbverspiegelte Scheibe gibt den Kabineninsassen ein Gefühl für das spätere Bild, ohne jedoch $\mathrm{zu}$ konkret $\mathrm{zu}$ werden. Trotz des beengenden Raumes entfalten diese Einschränkungen eine unglaubliche Kreativität der Nutzer.

Für die Gestaltung stellte sich die Frage: Ist es wirklich die Münze, die das Foto anstößt? Oder vielleicht ein Blick, ein Wort...? In der Kabine soll der Nutzer der Auslöser sein und den Moment der Aufnahme durch sein Verhalten rückgekoppelt steuern können.

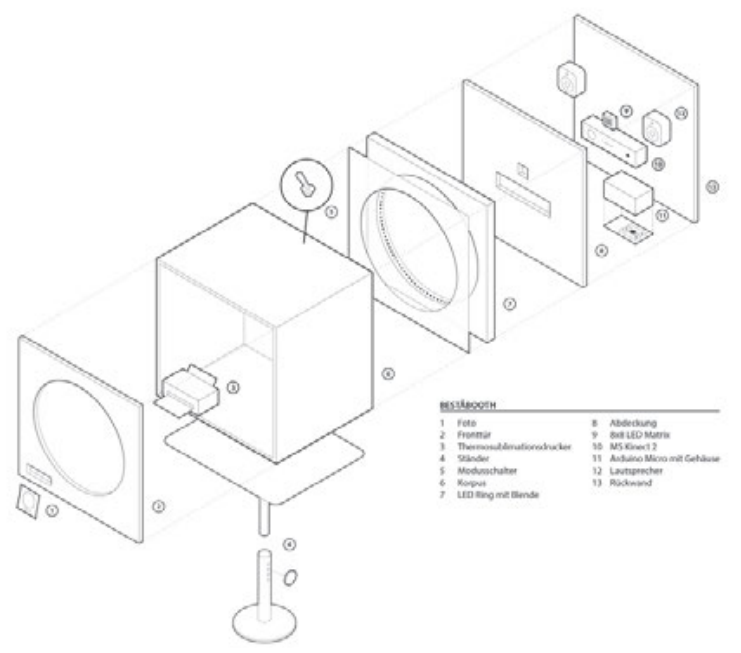

Abbildung 6: BESTABOOTH Explosion 
Die Nutzererkennung und Fotoaufnahme erfolgt mit einer Microsoft Kinect II. Durch die Vielzahl der inkludierten Sensoren (PrimeSense-Tiefensensor, HD Farbkamera) wird eine großzügige Abdeckung des Aktionsbereiches möglich. Niederkomplexe Icons als Handlungsunterstützung leiten den Nutzer und geben visuell Führung. Zwei Lautsprecher ergänzen den Output der Kabine auf akustischer Ebene.

Die Ansteuerung der Kinect erfolgt über ein MS Surface Pro Tablet, die der restlichen Komponenten über ein Arduino Micro Board. Software-seitig steuert eine ProcessingAnwendung die Logik der miteinander verketteten In- und Outputs.

Im aktuellen Stadium erkennt die Box einen oder mehrere Nutzer, die sich in ihrer Nähe aufhalten. Das Erkennen des Motives setzt eine Kaskade an Locksignalen mit multimodalen Affordanzen hervor, bis das Bild erstellt und als Print ausgegeben wird.

Im Einzelnen sind diese:

- $\quad$ Sehen - Visuell - LED Korona, LED Statusanzeige, Bildausgabe

- Hören - Auditiv - Lockrufe und Handlungsanweisungen

- Fühlen - Taktil - Randbegrenzung der Box spüren, Print aus Slot entnehmen

Das Activity Diagram zeigt sowohl die Eigenständigkeit der einzelnen Modalitäten, als auch ihre Verknüpfung untereinander.

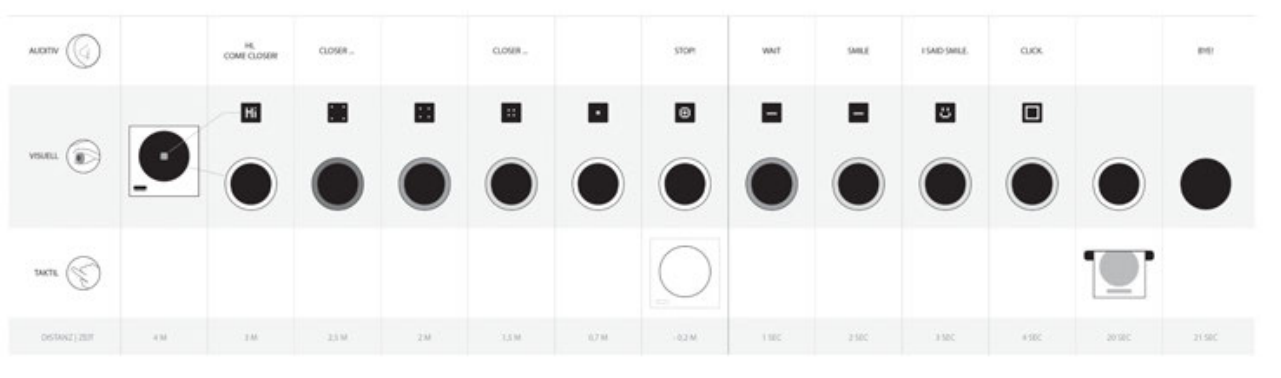

Abbildung 7: BESTÅBOOTH Activity Diagram

\section{Literatur}

Norman, D. (1989). Die Dinge des Alltags. Frankfurt/Main: Campus-Verlag.

Schmidt, R., Thews, G. \& Lang, F. (Hrsg.) (2000). Physiologie des Menschen. Heidelberg: Springer. 